ACS Chem Biol. 2016 July 15; 11(7): 1795-1799. doi:10.1021/acschembio.6b00037.

\title{
Identification of a Binding Site for Unsaturated Fatty Acids in the Orphan Nuclear Receptor Nurr1
}

\author{
Ian Mitchelle S. de Vera ${ }^{\dagger, \ddagger}$, Pankaj K. Giri ${ }^{\dagger, \ddagger}$, Paola Munoz-Tello $^{\dagger}$, Richard Brust ${ }^{\dagger}$, Jakob \\ Fuhrmann $^{\dagger}$, Edna Matta-Camacho ${ }^{\dagger}$, Jinsai Shang ${ }^{\dagger}$, Sean Campbell ${ }^{\dagger}$, Henry D. Wilson", \\ Juan Granados ${ }^{\S}$, William J. Gardner Jr${ }^{\S}$, Trevor P. Creamer", Laura. A. Solt ${ }^{\dagger}$, and Douglas J. \\ Kojetin ${ }^{\dagger,}{ }^{*}$ \\ †Department of Molecular Therapeutics, The Scripps Research Institute, Scripps Florida, Jupiter, \\ Florida 33458, USA \\ ITSRI Graduate Program, The Scripps Research Institute, Scripps Florida, Jupiter, Florida 33458, \\ USA
}

§TSRI High School Education Outreach Program; The Scripps Research Institute, Scripps Florida, Jupiter, Florida 33458, USA

"Center for Structural Biology, Department of Molecular and Cellular Biochemistry, University of Kentucky, Lexington, Kentucky 40536, USA

\begin{abstract}
Nurr1/NR4A2 is an orphan nuclear receptor, and currently there are no known natural ligands that bind Nurr1. A recent metabolomics study identified unsaturated fatty acids, including arachidonic acid and docosahexaenoic acid (DHA), that interact with the ligand-binding domain (LBD) of a related orphan receptor, Nur77/NR4A1. However, the binding location and whether these ligands bind other NR4A receptors were not defined. Here, we show that unsaturated fatty acids also interact with the Nurr1 LBD, and solution NMR spectroscopy reveals the binding epitope of DHA at its putative ligand-binding pocket. Biochemical assays reveal that DHA-bound Nurr1 interacts with high affinity with a peptide derived from PIAS $\gamma$, a protein that interacts with Nurr1 in cellular extracts, and DHA also affects cellular Nurr1 transactivation. This work is the first structural report of a natural ligand binding to a canonical NR4A ligand-binding pocket, and indicates a natural ligand can bind and affect Nurr1 function.
\end{abstract}

\section{Graphical abstract}

\footnotetext{
"Corresponding Author.dkojetin@scripps.edu.

*These authors contributed equally.

ASSOCIATED CONTENT

Supporting Information

Supporting Information includes methods and details of plasmids, ligands, protein expression and purification, LC-MS, tryptophan fluorescence spectroscopy, NMR, FP, TR-FRET, SEC, and cellular reporter assay; and additional figures. This information is available free of charge on the ACS Publications website.

The authors declare no competing financial interests.
} 


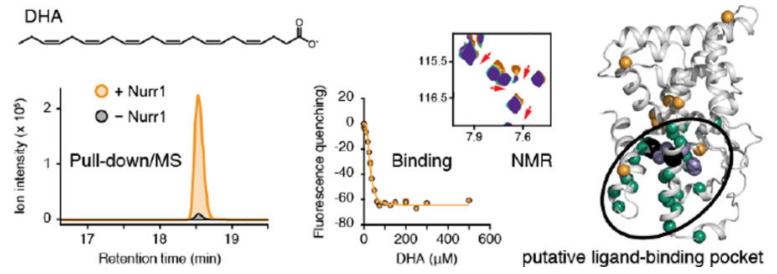

\section{Keywords}

Orphan nuclear receptor; NMR spectroscopy; ligand binding

Nuclear receptor (NR) transcription factors regulate many aspects of mammalian physiology, in part through binding small molecule ligands within a conserved "canonical" ligand-binding pocket located in the hydrophobic core of the ligand-binding domain (LBD). Ligand binding alters the LBD conformation and affects interaction of NR coregulators and other proteins resulting in alterations in chromatin and changes in gene expression. About half of the 48 human NRs were initially termed orphan NRs because their endogenous ligands were unknown. ${ }^{1,2}$ Some of these orphan NRs have since been "adopted" or "deorphanized" as natural ligands have been discovered. However, there remains a cohort of orphan NRs with unidentified natural ligands, including the NR4A receptors Nur77/ NR4A1, Nurr1/NR4A2, and NOR-1/NR4A3. The NR4As are expressed in many tissues, including skeletal muscle, adipose, cardiovascular, kidney, liver, and central nervous system, often with aberrant expression in disease with roles in inflammation, arthritis, cancer, neurological and metabolic conditions including obesity, dyslipidemia, diabetes, and cardiovascular disease. ${ }^{3-6}$

Crystal structures of the LBDs of Nurr1 and Nur77 show no apparent cavity in the canonical pocket where non-orphan nuclear receptors bind natural ligands. ${ }^{7}{ }^{8}$ Instead, their putative pockets are filled with bulky hydrophobic residues. Interestingly, a metabolomics study revealed that unsaturated fatty acids (UFAs) could be pulled down from Nur77 LBD protein incubated with a lipophilic metabolite extract from mouse brain and testes. ${ }^{9}$ This work was inspired by the discovery of synthetic Nur77 agonists that were later shown to bind to surface exposed alternate ligand-binding sites. ${ }^{10,11}$ Analysis of two UFAs identified as the most abundantly enriched in the metabolomics workflow, arachidonic acid (AA) and docosahexaenoic acid (DHA), confirmed direct binding to Nur77. However, the structural basis of the UFA-Nur77 interaction, and whether UFAs can bind other NR4A receptors such as Nurr1, remain elusive.

To determine if Nurr1 can bind DHA, we performed a pull-down assay (Figure 1A) by incubating DHA with polyhistidine-tagged (6xHis) Nurr1 LBD bound to $\mathrm{Ni}^{2+}$-NTA resin beads, or as a control with $\mathrm{Ni}^{2+}{ }_{-} \mathrm{NTA}$ resin alone to account for background binding to the resin. After washing the resin, methanol was added to elute any bound DHA and the elution fraction was analyzed by liquid chromatography-mass spectrometry (LC-MS). A 25-fold enrichment of DHA was observed for Nurr1 LBD over control (Figure 1B). High-resolution mass spectrometry confirmed the identity of the LC peak as DHA (Figure 1C). These data 
indicate that Nurr1 LBD is able to selectively bind and enrich DHA to a similar extent as Nur77. 9

Nurr1 contains two tryptophan residues located in the LBD core and putative ligand-binding pocket. To confirm the LC-MS enrichment results and to test the direct interaction between Nurr1 LBD and DHA, we performed steady-state tryptophan fluorescence spectroscopy. Addition of DHA to Nurr1 LBD decreased tryptophan fluorescence emission demonstrating direct binding (Figure 2A). Furthermore, titration of the UFAs DHA and AA, but not the saturated fatty acid stearic acid (SA), caused a concentration-dependent decrease in fluorescence (Figure $2 \mathrm{~B}) . \mathrm{K}_{\mathrm{d}}$ values of DHA $(30 \pm 1 \mu \mathrm{M})$ and AA $(58 \pm 10 \mu \mathrm{M})$ to Nurr1 are similar to DHA binding to another NR known to bind DHA, RXRa (Supporting Information, Figure $\mathrm{S} 1 ; \mathrm{K}_{\mathrm{d}}=33 \pm 2 \mu \mathrm{M}$ ). These data indicate Nurr1 selectively binds UFAs, as observed for Nur77. ${ }^{9}$

Because the ligand-free/apo-Nur77 crystal structure lacks space for a ligand to bind within its putative canonical ligand-binding pocket, it was postulated that UFAs may bind to a nontraditional pocket. ${ }^{9}$ For example, two non-canonical, alternate ligand-binding sites were observed on the surface of Nur77 in crystal structures bound to synthetic cytosporone B analogs. ${ }^{12-14}$ Because our efforts to obtain a co-crystal structure of the DHA-Nurr1 LBD complex have not yet succeeded, we used solution NMR spectroscopy to map the structural binding epitope of DHA. Titration of DHA into ${ }^{15} \mathrm{~N}$-labeled Nurr1 LBD resulted in NMR peak shifting and reduction in peak intensity due to NMR resonance line broadening for specific peaks indicating localized binding effects (Figure 3A and Supporting Information, Figure S2). Line broadening here is a result of chemical exchange between apo and DHAbound states due to the $\mu \mathrm{M}$ binding affinity, as well as changes in Nurr1 motions (dynamics) when bound to DHA. ${ }^{15}$ DHA binding also affects methyl groups buried in the Nurr1 LBD core and putative pocket (Figure 3B; Leu410, Ile483, and Ile486), and similar effects are observed for AA binding to Nurr1 LBD (Supporting Information, Figure S3). In contrast, addition of SA, which does not bind Nurr1 as observed by tryptophan fluorescence (Figure 2B), revealed no NMR perturbations (Supporting Information, Figure S4).

To determine the location of DHA binding, we quantitated 2D NMR peak intensity changes and chemical shift perturbations (CSPs) (Figure 3C,D). Mapping these data onto the apoNurr1 LBD crystal structure ${ }^{7}$ revealed that residues with the largest NMR perturbations localize to the putative ligand-binding pocket (Figure 3E). The perturbations are not widespread indicating DHA binding to Nurr1 preserves the overall LBD structure. Notably, NMR analysis of ligand binding to the canonical pocket of other NRs, including RXRa, PPAR $\gamma$, and VDR similarly causes perturbations for residues in the interior core of the LBD (i.e., the canonical ligand-binding pocket). ${ }^{16-22}$ By comparison, our NMR data on the DHA-Nurr1 interaction indicate that UFAs bind to the putative canonical ligand-binding pocket in Nurr1. Furthermore, DHA binding also perturbs NMR peaks of residues in the C-terminal helix 12 (e.g., Figure 3A; Ile588, Lys590), a region important for NR LBD-coregulator protein interaction.

Comparison of our DHA-Nurr1 NMR interaction data to available crystal structures of Nur77 bound to cytosporone B agonist analogs ${ }^{12-14}$ indicates that UFAs do not bind to 
Nurr1 at the same surface-exposed alternate sites (Supporting Information, Figure S5). Furthermore, unlike UFA binding to Nur77, which stabilized an oligomer species, ${ }^{9}$ DHA binding to Nurr1 shifts the monomer-dimer equilibrium in favor of monomer (Supporting Information, Figure S6) resulting in one relatively modest CSP at the putative helix 11 dimer interface (Figure 3D,E; i.e., Asn547) indicating no substantial conformational change to this surface. However, CSPs to the Nurr1 ligand-binding pocket are more numerous. Notably, the DHANurr1 NMR interaction epitope is similar to the NMR interaction epitope recently mapped for amodiaquine, a synthetic Nurr1 agonist that binds Nurr1, ${ }^{23}$ indicating overlapping natural and synthetic ligand-binding sites.

We next determined if an UFA binding to Nurr1 LBD could affect Nurr1 function. PIAS $\gamma$ is a SUMO-E3 ligase and coregulator of Nurr1 that was previously shown to bind Nurr1 LBD in whole cell extracts through an essential LXXLL coregulator motif sequence and repress Nurr1 transactivation. ${ }^{24}$ We developed a fluorescence polarization (FP)-based biochemical assay using a peptide derived from the essential PIAS $\gamma$ LXXLL motif. Increasing amounts of Nurr1 LBD were added to a fixed concentration of the FITC-labeled PIAS $\gamma$ peptide (Figure 4A). In the absence of DHA, we observed very weak interaction with the PIAS $\gamma$ peptide. However, in the presence of DHA, a robust interaction is observed between Nurr1 LBD and the FITC-PIAS $\gamma$ peptide $\left(\mathrm{K}_{\mathrm{d}}=3.5 \pm 0.8 \mu \mathrm{M}\right)$. To confirm these data using an orthogonal method, we developed a time-resolved fluorescence resonance energy transfer (TR-FRET) assay. Titration of DHA resulted in a concentration-dependent increase in the interaction between Nurr1 LBD and the FITCPIAS $\gamma$ peptide, confirming that DHA promotes the interaction with an $\mathrm{EC}_{50}$ of $25 \pm 1.2 \mu \mathrm{M}$ (Figure $5 \mathrm{~B}$ ), consistent with the $30 \mu \mathrm{M}$ $\mathrm{K}_{\mathrm{d}}$ determined by tryptophan fluorescence (Figure 2B).

To determine if DHA could affect Nurr1 transactivation, we used a cell-based luciferase reporter gene cotransfection assay that reports on transcription occurring from direct binding to its cognate DNA response element. We performed this assay in two cell lines, HEK293T (a general, commonly used cell line) and MN9D (used as a dopaminergic cellular model related to Nurr1 function). An expression plasmid encoding full-length Nurr1 was cotransfected with a reporter plasmid containing three copies of the NR4A monomeric DNA-response element (3xNBRE) upstream of the firefly luciferase gene Transfection of full-length Nurr1, but not an empty vector control expression plasmid, resulted in a significant increase in luciferase activity (Figure 4C). This demonstrates the 3xNBREluciferase reporter is specifically activated by Nurr1 overexpression. Cellular treatment with DHA and Nurr1 overexpression caused a statistically significant decrease in luciferase activity (Figure 4D) relative to the significant activation caused by Nurr1 overexpression alone (Figure 4C), indicating DHA can affect Nurr1 transactivation.

In conclusion, here we reveal that Nurr1 directly binds UFAs, which is the first demonstration of a natural ligand binding to Nurr1. This observation adds to the complex polypharmacology of UFAs, which bind other non-NR proteins and other NRs including RXRs, Nur77, FXR, LXRs, and PPARs, and affect their function also at $\mu \mathrm{M}$ concentrations. Our tryptophan fluorescence binding analysis shows that the affinity of DHA for Nurr1 and $\mathrm{RXRa}$ is essentially the same ( $30 \mu \mathrm{M}$ and $33 \mu \mathrm{M}$, respectively). This observation, combined with our solution NMR studies that map the structural binding epitope of DHA to the 
putative Nurr1 ligand- binding pocket, indicates DHA binding to Nurr1 is specific.

Furthermore, given the high presence of UFAs and in particular DHA in tissues where Nurr1 is present, such as the brain and retina (40\% and $60 \%$ of total UFAs, respectively), they are poised to potentially bind Nurr1 in vivo. One point to note is that our data show that DHA binding favors a monomeric LBD over a dimer species. However, the dimer species is of low abundance and may probably have low affinity, but this relatively modest equilibrium change could in principle have an effect on Nurr1 function. Another point to note is that it is possible that sub-stoichiometric amounts of a bacterial endogenous ligand bound to Nurr1 could in principle influence the binding studies. However, using NMR we have observed that subjecting DHA-bound Nurr1 to dialysis results in an NMR spectrum nearly identical to freshly purified Nurr1 (Supporting Information, Figure S7). Thus if there is an endogenous ligand bound to Nurr1 in bacteria, it likely dissociates out of the pocket resulting during purification in apo/ligand-free protein. These data also demonstrate that DHA-binding to the Nurr1 LBD is reversible.

Furthermore, our data also show that DHA-bound Nurr1 LBD, but not apo-Nurr1 LBD, binds with high affinity to an LXXLL peptide derived from PIAS $\gamma$ that essential for interaction with Nurr1.$^{24}$ The modest but statistically significant effect of DHA on Nurr1 transactivation could be the result of several consequences. It could be that DHA binding to Nurr1 may not substantially affect or perturb the already high cellular activation of DNA binding-dependent Nurr1 transcription. This could be the case whether or not Nurr1 binds an endogenous ligand present in cells. However, if there is a hypothetical endogenous ligand that binds and activates Nurr1 in cells, DHA may not compete with it for binding Nurr1. Finally, because PIAS $\gamma$ can SUMOylate Nurr1 independent of its ability to repress Nurr1 transctivation, ${ }^{24,} 25$ future work is needed to explore roles of UFA binding in PIAS $\gamma$ mediated functions of Nurr1 and other cellular outcomes such as gene expression.

Apo-Nurr1 and Nur77 crystal structures show no canonical binding pocket to which a natural ligand can bind as they have $\sim 30-40 \AA^{3}$ putative pocket volumes. ${ }^{26}$ However, structural studies have shown that the ligand-binding pockets of some adopted orphan NRs can undergo large conformational changes to bind natural ligands. A crystal structure of apoREV-ERB revealed a collapsed pocket with no ligand-binding cavity $\left(\sim 50-90 \AA^{3}\right) .{ }^{27}$ However, a subsequent crystal structure revealed its pocket undergoes a large expansion to bind the porphyrin heme $\left(\sim 600 \AA^{3}\right) .{ }^{28}$ NMR studies revealed that the apo-REV-ERB ligandbinding pocket is dynamic on the $\mu \mathrm{s}$-ms timescale, ${ }^{29}$ confirming the collapsed apo-pocket captured by crystallography has the ability to expand in solution as part of the structural mechanism of binding heme. A previous NMR study on Nurr1 showed that residues comprising its putative ligand-binding pocket have shorter $T_{2}$ relaxation times indicating motion on the $\mu$ s-ms NMR time-scale. ${ }^{30}$ Like the REV-ERBs, this motion might be critical for the large conformational changes required for ligand binding to Nurr1, and future structural studies including crystallography are needed to reveal the molecular details of UFA binding to Nurr1.

\section{Supplementary Material}

Refer to Web version on PubMed Central for supplementary material. 


\section{Acknowledgments}

We thank M. Gill (NCI/NIH) for assistance with Python scripts; A. Heller and L. Won (U. Chicago), and M. Zigmond and J. Jaumotte (U. Pittsburgh) for providing MN9D cells; B. Shen (TSRI) for providing access to the high-resolution mass spectrometer; and P. Griffin (TSRI) for valuable input.

Funding Sources

Research reported in this publication was supported by the National Institute of General Medical Sciences (NIGMS) of the National Institutes of Health (NIH) under award number R01GM114420. The content is solely the responsibility of the authors and does not necessarily represent the official views of the NIH.

\section{ABBREVIATIONS}

Nurr1 nuclear receptor related 1 protein

NMR nuclear magnetic resonance spectroscopy

DHA docosahexaenoic acid

AA arachidonic acid

SA stearic acid

LBD ligand-binding domain

UFA unsaturated fatty acids

Nur77 nerve growth factor IB

NOR-1 neuron-derived orphan receptor 1

LC-MS liquid chromatography-mass spectrometry

FITC Fluorescein isothiocyanate

RXR retinoid $\mathrm{X}$ receptor

PIAS $\gamma \quad$ protein inhibitor of activated STAT protein gamma

\section{REFERENCES}

1. Chawla A, Repa JJ, Evans RM, Mangelsdorf DJ. Nuclear receptors and lipid physiology: opening the X-files. Science. 2001; 294:1866-1870. [PubMed: 11729302]

2. O'Malley B. The steroid receptor superfamily: more excitement predicted for the future. Mol Endocrinol. 1990; 4:363-369. [PubMed: 2188115]

3. Maxwell MA, Muscat GE. The NR4A subgroup: immediate early response genes with pleiotropic physiological roles. Nuclear receptor signaling. 2006; 4:e002. [PubMed: 16604165]

4. Pearen MA, Muscat GE. Minireview: Nuclear hormone receptor 4A signaling: implications for metabolic disease. Mol Endocrinol. 2010; 24:1891-1903. [PubMed: 20392876]

5. McMorrow JP, Murphy EP. Inflammation: a role for NR4A orphan nuclear receptors? Biochemical Society transactions. 2011; 39:688-693. [PubMed: 21428963]

6. Beard JA, Tenga A, Chen T. The interplay of NR4A receptors and the oncogene-tumor suppressor networks in cancer. Cell Signal. 2015; 27:257-266. [PubMed: 25446259] 
7. Wang Z, Benoit G, Liu J, Prasad S, Aarnisalo P, Liu X, Xu H, Walker NP, Perlmann T. Structure and function of Nurr1 identifies a class of ligand-independent nuclear receptors. Nature. 2003; 423:555560. [PubMed: 12774125]

8. Flaig R, Greschik H, Peluso-Iltis C, Moras D. Structural basis for the cell-specific activities of the NGFI-B and the Nurr1 ligand-binding domain. J Biol Chem. 2005; 280:19250-19258. [PubMed: 15716272]

9. Vinayavekhin N, Saghatelian A. Discovery of a protein-metabolite interaction between unsaturated fatty acids and the nuclear receptor Nur77 using a metabolomics approach. J Am Chem Soc. 2011; 133:17168-17171. [PubMed: 21973308]

10. Cho SD, Lee SO, Chintharlapalli S, Abdelrahim M, Khan S, Yoon K, Kamat AM, Safe S. Activation of nerve growth factor-induced $\mathrm{B}$ alpha by methylene-substituted diindolylmethanes in bladder cancer cells induces apoptosis and inhibits tumor growth. Molecular pharmacology. 2010; 77:396-404. [PubMed: 20023005]

11. Zhan Y, Du X, Chen H, Liu J, Zhao B, Huang D, Li G, Xu Q, Zhang M, Weimer BC, Chen D, Cheng Z, Zhang L, Li Q, Li S, Zheng Z, Song S, Huang Y, Ye Z, Su W, Lin SC, Shen Y, Wu Q. Cytosporone B is an agonist for nuclear orphan receptor Nur77. Nat Chem Biol. 2008; 4:548-556. [PubMed: 18690216]

12. Li L, Liu Y, Chen HZ, Li FW, Wu JF, Zhang HK, He JP, Xing YZ, Chen Y, Wang WJ, Tian XY, Li AZ, Zhang Q, Huang PQ, Han J, Lin T, Wu Q. Impeding the interaction between Nur77 and p38 reduces LPS-induced inflammation. Nat Chem Biol. 2015; 11:339-346. [PubMed: 25822914]

13. Wang WJ, Wang Y, Chen HZ, Xing YZ, Li FW, Zhang Q, Zhou B, Zhang HK, Zhang J, Bian XL, Li L, Liu Y, Zhao BX, Chen Y, Wu R, Li AZ, Yao LM, Chen P, Zhang Y, Tian XY, Beermann F, Wu M, Han J, Huang PQ, Lin T, Wu Q. Orphan nuclear receptor TR3 acts in autophagic cell death via mitochondrial signaling pathway. Nat Chem Biol. 2014; 10:133-140. [PubMed: 24316735]

14. Zhan YY, Chen Y, Zhang Q, Zhuang JJ, Tian M, Chen HZ, Zhang LR, Zhang HK, He JP, Wang WJ, Wu R, Wang Y, Shi C, Yang K, Li AZ, Xin YZ, Li TY, Yang JY, Zheng ZH, Yu CD, Lin SC, Chang C, Huang PQ, Lin T, Wu Q. The orphan nuclear receptor Nur77 regulates LKB1 localization and activates AMPK. Nat Chem Biol. 2012; 8:897-904. [PubMed: 22983157]

15. Kleckner IR, Foster MP. An introduction to NMR-based approaches for measuring protein dynamics. Biochimica et biophysica acta. 2011; 1814:942-968. [PubMed: 21059410]

16. Lu J, Cistola DP, Li E. Analysis of ligand binding and protein dynamics of human retinoid X receptor alpha ligand-binding domain by nuclear magnetic resonance. Biochemistry. 2006; 45:1629-1639. [PubMed: 16460010]

17. Johnson BA, Wilson EM, Li Y, Moller DE, Smith RG, Zhou G. Ligand-induced stabilization of PPARgamma monitored by NMR spectroscopy: implications for nuclear receptor activation. J Mol Biol. 2000; 298:187-194. [PubMed: 10764590]

18. Hughes TS, Chalmers MJ, Novick S, Kuruvilla DS, Chang MR, Kamenecka TM, Rance M, Johnson BA, Burris TP, Griffin PR, Kojetin DJ. Ligand and receptor dynamics contribute to the mechanism of graded PPAR $\gamma$ agonism. Structure. 2012; 20:139-150. [PubMed: 22244763]

19. Singarapu KK, Zhu J, Tonelli M, Rao H, Assadi-Porter FM, Westler WM, DeLuca HF, Markley JL. Ligand-specific structural changes in the vitamin D receptor in solution. Biochemistry. 2011; 50:11025-11033. [PubMed: 22112050]

20. Lu J, Dawson MI, Hu QY, Xia Z, Dambacher JD, Ye M, Zhang XK, Li E. The effect of antagonists on the conformational exchange of the retinoid $\mathrm{X}$ receptor alpha ligand-binding domain. Magn Reson Chem. 2009; 47:1071-1080. [PubMed: 19757405]

21. Hughes TS, Giri PK, de Vera IM, Marciano DP, Kuruvilla DS, Shin Y, Blayo AL, Kamenecka TM, Burris TP, Griffin PR, Kojetin DJ. An alternate binding site for PPAR $\gamma$ ligands. Nature communications. 2014; 5:3571.

22. Berger JP, Petro AE, Macnaul KL, Kelly LJ, Zhang BB, Richards K, Elbrecht A, Johnson BA, Zhou G, Doebber TW, Biswas C, Parikh M, Sharma N, Tanen MR, Thompson GM, Ventre J, Adams AD, Mosley R, Surwit RS, Moller DE. Distinct properties and advantages of a novel peroxisome proliferator-activated protein [gamma] selective modulator. Mol Endocrinol. 2003; 17:662-676. [PubMed: 12554792] 
23. Kim CH, Han BS, Moon J, Kim DJ, Shin J, Rajan S, Nguyen QT, Sohn M, Kim WG, Han M, Jeong I, Kim KS, Lee EH, Tu Y, Naffin-Olivos JL, Park CH, Ringe D, Yoon HS, Petsko GA, Kim KS. Nuclear receptor Nurr1 agonists enhance its dual functions and improve behavioral deficits in an animal model of Parkinson's disease. Proc Natl Acad Sci U S A. 2015; 112:8756-8761. [PubMed: 26124091]

24. Arredondo C, Orellana M, Vecchiola A, Pereira LA, Galdames L, Andres ME. PIASgamma enhanced SUMO-2 modification of Nurr1 activation-function-1 domain limits Nurr1 transcriptional synergy. PloS one. 2013; 8:e55035. [PubMed: 23358114]

25. Galleguillos D, Vecchiola A, Fuentealba JA, Ojeda V, Alvarez K, Gomez A, Andres ME. PIASgamma represses the transcriptional activation induced by the nuclear receptor Nurr1. J Biol Chem. 2004; 279:2005-2011. [PubMed: 14559918]

26. Gallastegui N, Mackinnon JA, Fletterick RJ, Estebanez-Perpina E. Advances in our structural understanding of orphan nuclear receptors. Trends in biochemical sciences. 2015; 40:25-35. [PubMed: 25499868]

27. Woo EJ, Jeong DG, Lim MY, Jun Kim S, Kim KJ, Yoon SM, Park BC, Ryu SE. Structural insight into the constitutive repression function of the nuclear receptor Rev-erbbeta. J Mol Biol. 2007; 373:735-744. [PubMed: 17870090]

28. Pardee KI, Xu X, Reinking J, Schuetz A, Dong A, Liu S, Zhang R, Tiefenbach J, Lajoie G, Plotnikov AN, Botchkarev A, Krause HM, Edwards A. The structural basis of gas-responsive transcription by the human nuclear hormone receptor REV-ERBbeta. PLoS Biol. 2009; 7:e43. [PubMed: 19243223]

29. Matta-Camacho E, Banerjee S, Hughes TS, Solt LA, Wang Y, Burris TP, Kojetin DJ. Structure of REV-ERBbeta ligand-binding domain bound to a porphyrin antagonist. J Biol Chem. 2014; 289:20054-20066. [PubMed: 24872411]

30. Michiels P, Atkins K, Ludwig C, Whittaker S, van Dongen M, Gunther U. Assignment of the orphan nuclear receptor Nurr1 by NMR. Biomol NMR Assign. 2010; 4:101-105. [PubMed: 20300892] 


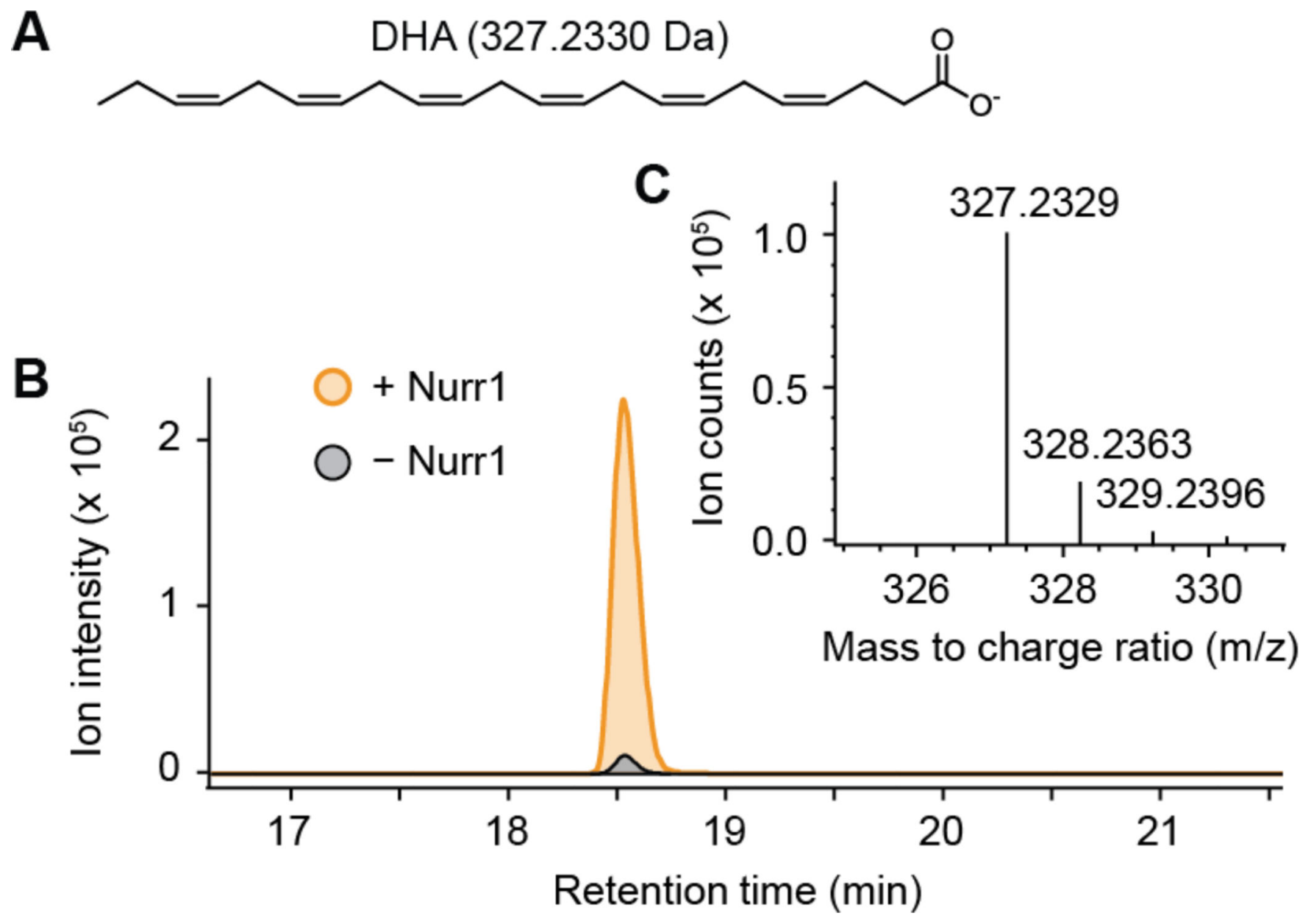

Figure 1.

DHA pull-down in the presence of 6xHis-Nurr1 LBD. (A) Structure of DHA. (B) LC-MS shows enrichment of DHA in the presence of Nurr1 LBD. (C) High-resolution MS confirmation of DHA. 


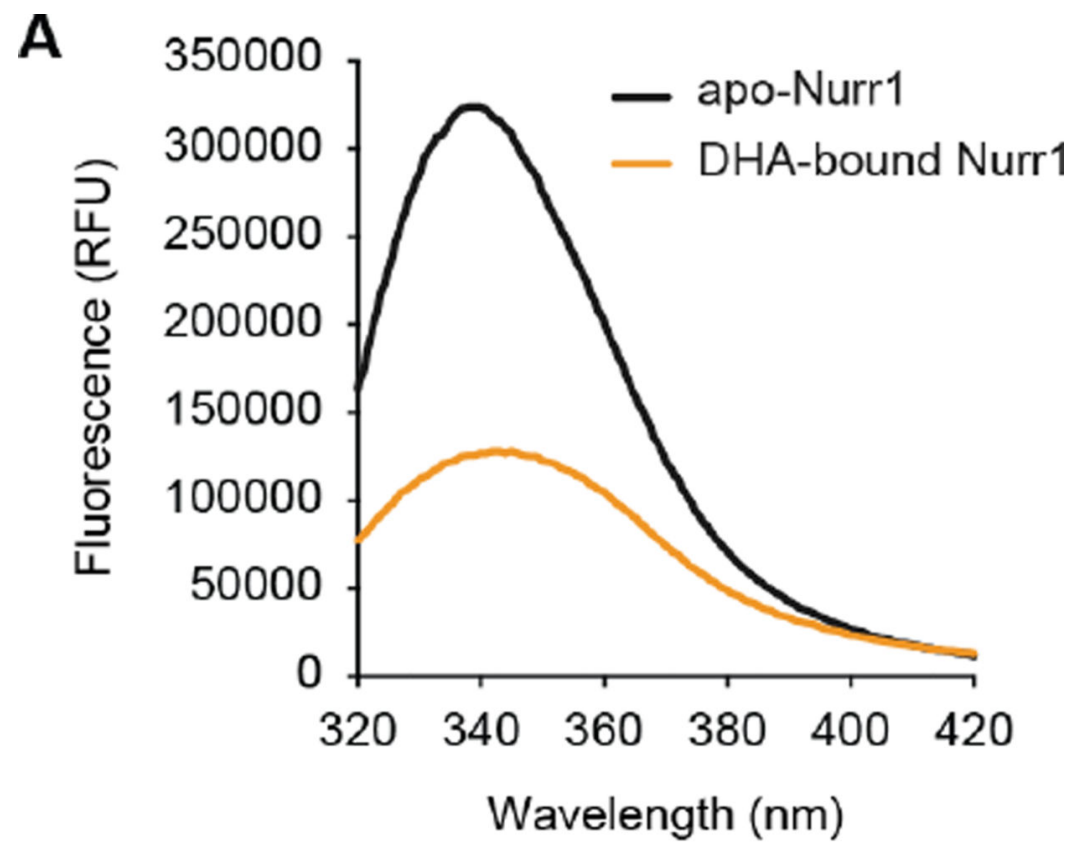

B

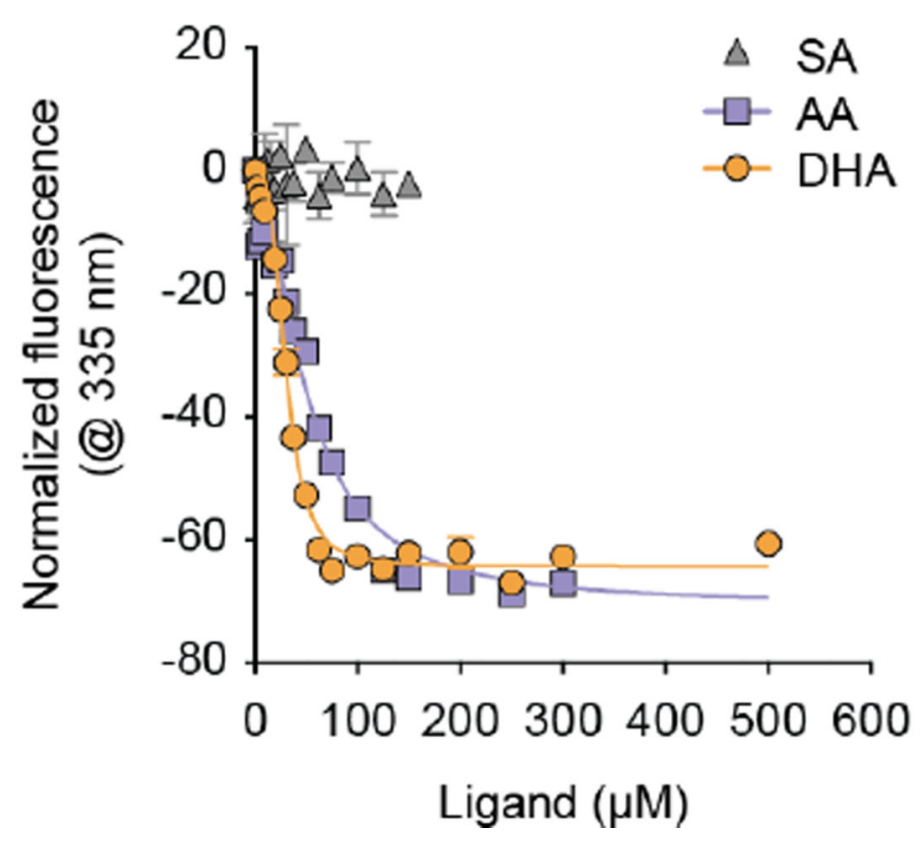

Figure 2.

Binding of UFAs to Nurr1 LBD. (A) Tryptophan fluorescence emission spectra of Nurr1 LBD \pm DHA. (B) Titration of DHA and AA, but not SA, decreases Nurr1 fluorescence. 
A
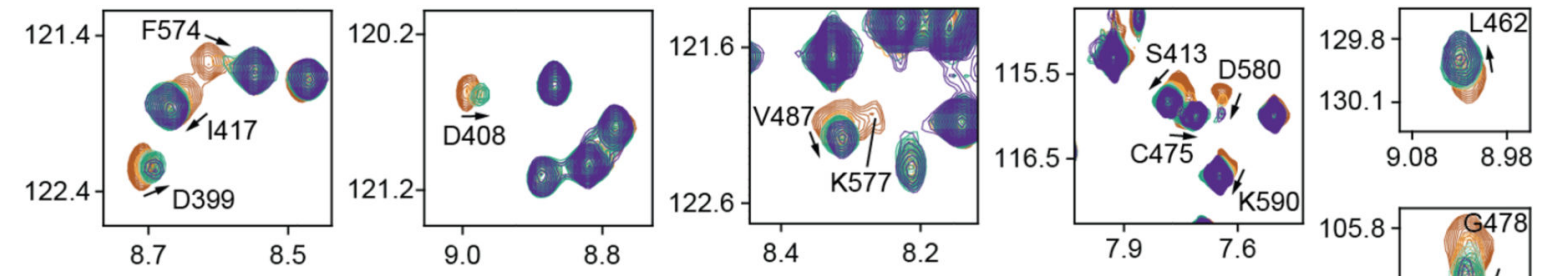

Vehicle

$0.4 \times$ DHA
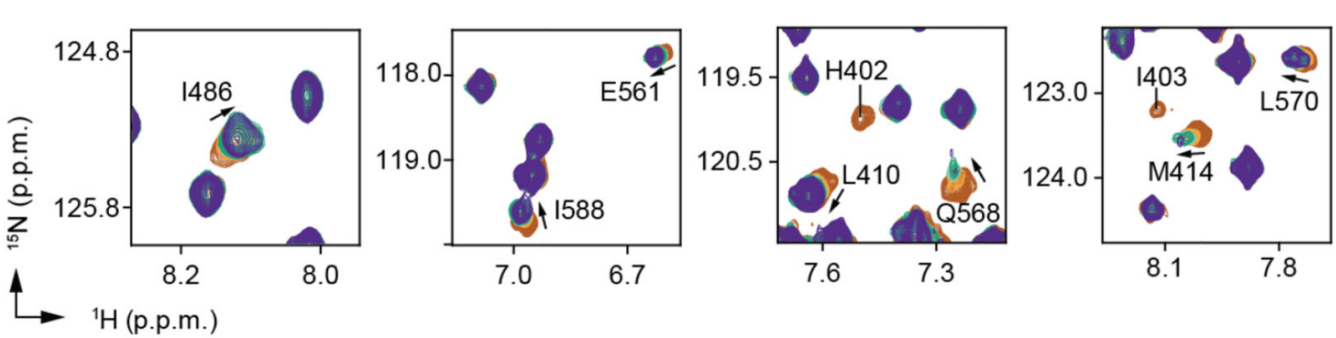
106.1

$0.8 \times$ DHA

$1.5 \times \mathrm{DHA}$

B

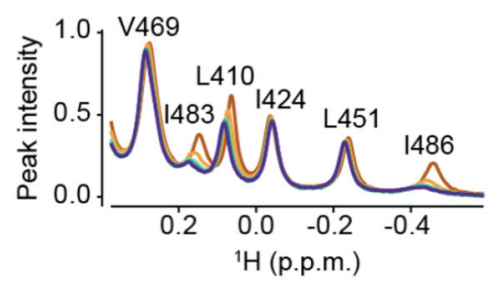

C

E

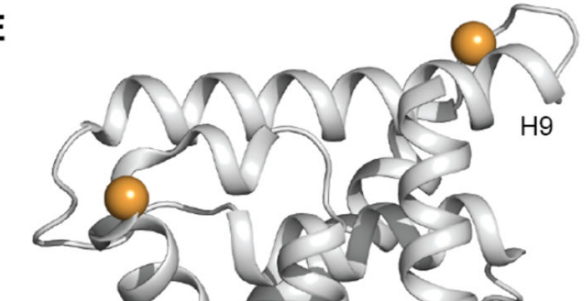

D
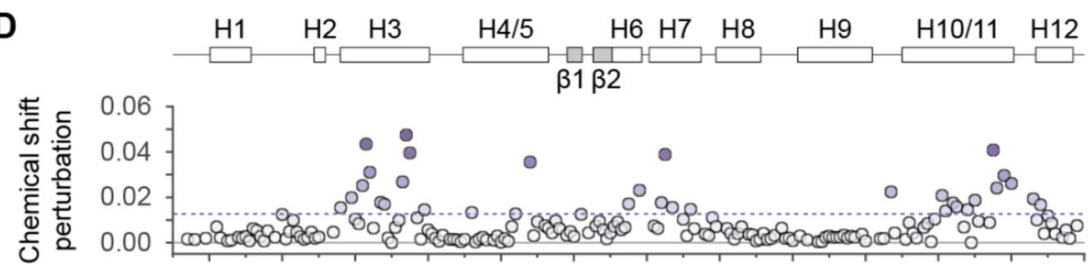

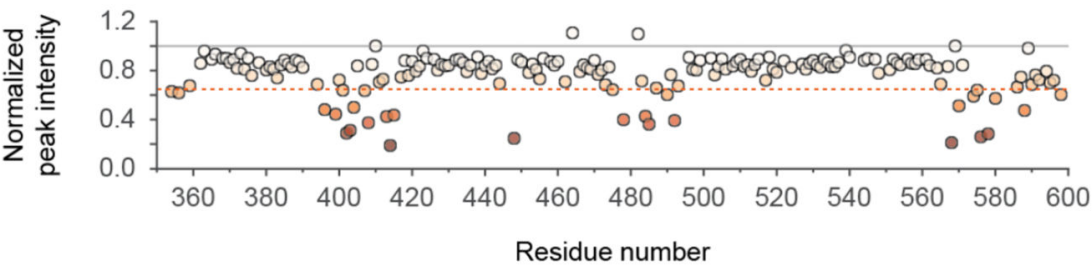

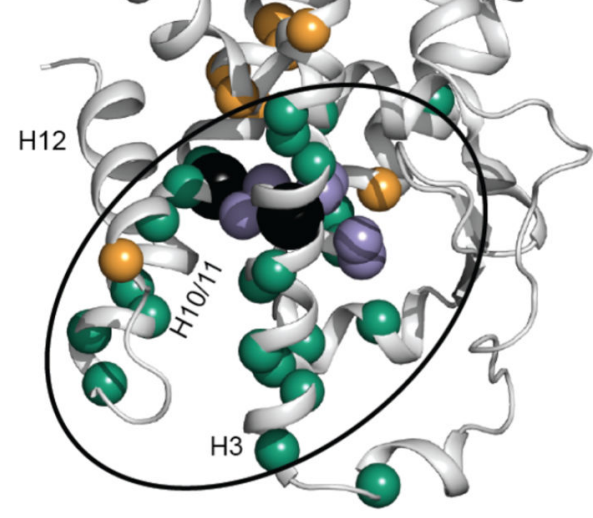

putative ligand-binding pocket

Figure 3.

NMR analysis of DHA binding to Nurr1. (A,B) DHA titration followed by $2 \mathrm{D}\left[{ }^{1} \mathrm{H},{ }^{15} \mathrm{~N}\right]-$

TROSY-HSQC NMR of backbone amides and (B) 1D [ $\left.{ }^{1} \mathrm{H}\right]-$ NMR data of the methyl region. (C) Representative chemical shift perturbation (CSP). (D) Quantitation of 2D NMR peak intensities and CSPs. (E) NMR data mapped on the apo-Nurr1 LBD (PDB 1OVL) ${ }^{7}$. Spheres show residues with changes beyond dotted line thresholds in (D); backbone amides that disappear (black) or are perturbed (CSP only, orange; CSP and intensity, teal); methyl peaks perturbed (purple). 

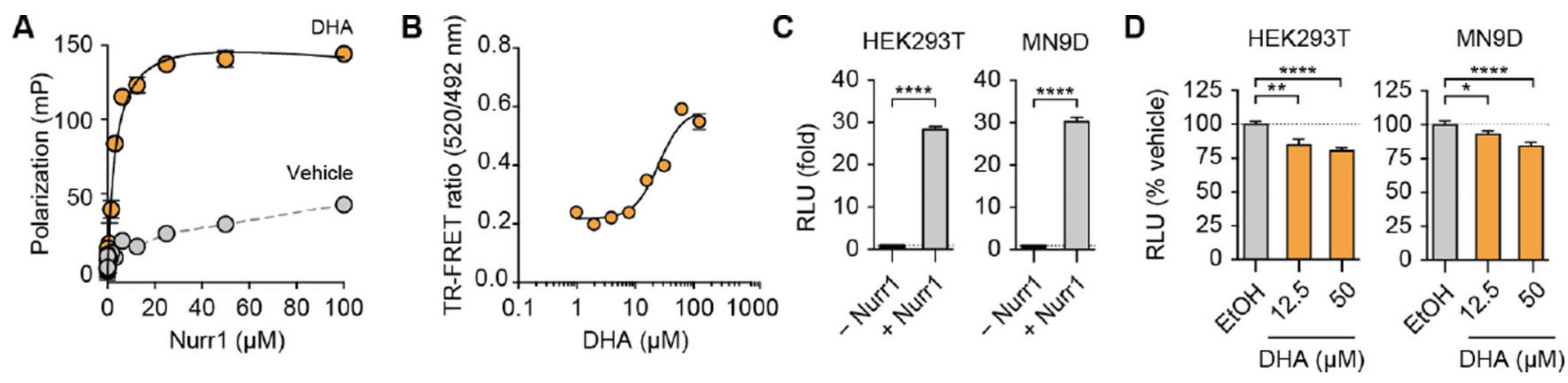

Figure 4.

Effect of DHA on Nurr1 function. (A) Fluorescence polarization (FP) assay shows increased binding of PIAS $\gamma$ peptide to Nurr1 LBD in the presence of DHA. (B) Time-resolved fluorescence resonance energy transfer (TR-FRET) assay shows a DHA concentrationdependent increase in the binding of PIAS $\gamma$ peptide to Nurr1 LBD. (C,D) Cell-based luciferase reporter gene assay analysis of Nurr1 transcription using a 3xNBRE-luciferase reporter. (C) Cells cotransfected with 3xNBRE reporter, with or without a full-length Nurr1 expression plasmid. (D) Cells cotransfected with 3xNBRE and a full-length Nurr1 expression plasmid treated with DHA or vehicle control (ethanol; EtOH). 